\title{
TMek: UN MICROCHIP PER DIAGNOSTICARE LA MALARIA IN MODO QUANTITATIVO E RAPIDO
}

\author{
RICCARDO BERTACCO (*), FRANCESCA MILESI (*), \\ PIETRO LORENZO COPPADORO $(* *)$, MARCO GIACOMETTI $(* *)$, \\ GIANFRANCO BENIAMINO FIORE (**), GIORGIO FERRARI (**)
}

Nota presentata dal Riccardo Bertacco e Marino Gatto

(Adunanza del 6 febbraio 2020)

\begin{abstract}
SuNTO. - La malaria è ancora una delle più importanti malattie infettive trasmessa dalle zanzare, con 3.5 miliardi di persone ancora a rischio, 229 milioni di nuovi casi e 409.000 morti solo nel 2019. L'infezione è causata da un parassita (Plasmodio) che attacca i globuli rossi nutrendosi di emoglobina e trasformandola in nanocristalli di emozoina. In questo lavoro si descrive un nuovo test pan-malarico (TMek), che sfrutta le proprietà paramagnetiche dei nanocristalli di emozoina per implementare su un chip la quantificazione automatica dei globuli rossi infetti. Sfruttando la competizione tra gravità e forze magnetiche, i globuli rossi infetti contenuti in un campione di sangue intero vengono catturati su concentratori micromagnetici e rilevati elettricamente, consentendo la misura della loro concentrazione con un limite di sensibilità fino a 10 parassiti/ $\mu$ l.
\end{abstract}

$* * *$

ABSTRACT. - Despite the huge efforts for malaria eradication, this infectious disease still represents a critical issue worldwide, with 3.5 billion people still at risk, 229 million new cases and 409.000 deaths in 2019. The infection is caused by the Plasmodiun parasite which attacks red blood cells, feeds on hemoglobin and transform it into hemozoin nanocrystals. In this paper we report on a novel pan-malaria test (TMek), based on the paramagnetic properties of hemozoin nanocrystals, which allows for the automatic quantification of infected red blood cells (i-RBCs) on a microchip. Exploiting the

(*) Dipartimento di Fisica, Politecnico di Milano, Milano, Italy.

E-mail: riccardo.bertacco@polimi.it

Dipartimento di Elettronica Informatica e Bioingegneria, Politecnico di Milano, Milano, Italy 
competition between gravity and magnetic forces, i-RBCs in a whole blood specimen are captured on micromagnetic concentrators and electrically detected, allowing for the measurement of their concentration with a limit of sensitivity down to 10 parasites/ $\mu$ l.

\section{INTRODUZIONE}

Nonostante gli ingenti sforzi profusi per sconfiggere la malaria, ${ }^{[1,2,3]}$ questa infezione rappresenta ancora una delle principali cause di morbidità e mortalità in tutto il mondo, con il più elevato numero di decessi registrato tra i bambini nell'Africa subsahariana. ${ }^{[4]} \mathrm{Al}$ giorno d'oggi è chiaro che per sconfiggere la malaria, è necessario un approccio integrato su diversi fronti: sviluppo di nuovi vaccini, ${ }^{[5,6]}$ nuovi farmaci antimalarici, metodi per bloccare la trasmissione della malaria e nuovi strumenti diagnostici. ${ }^{[7]}$ Per quanto riguarda la diagnostica, anche se l'Organizzazione mondiale della sanità (OMS) raccomanda vivamente di effettuare una diagnosi accurata prima di somministrare farmaci antimalarici, nella pratica un metodo diagnostico semplice, efficace e impiegabile sul campo non è ancora disponibile. L'analisi microscopica, il metodo di riferimento tradizionale nella diagnostica della malaria, è incompatibile con un ampio screening della popolazione in zone endemiche a causa dei lunghi tempi di esecuzione, della dipendenza dei risultati dall'operatore e della necessità di un microscopio ottico di buona qualità. ${ }^{[8]}$ La sensibilità ovviamente dipende dall'operatore che deve riconoscere i globuli rossi infetti (i-RBC) in circa 200 campi di osservazione, e si attesta intorno a 20 parassiti/ $\mu$ l. I test diagnostici rapidi (RTD) basati sulla rilevazione degli antigeni hanno rappresentato una svolta, in quanto consentono di ridurre il tempo di esecuzione dai 30-60 minuti della microscopia a circa 15 minuti e non richiedono un operatore qualificato. Essi hanno però un limite di sensibilità (LoD) relativamente alto, pari a circa 200 parassiti/ $\mu$ l, sono puramente qualitativi e non fanno distinzione tra infezioni passate e in atto. ${ }^{[9]}$ Inoltre, le prestazioni non ottimali degli $\mathrm{RDT}$ per il rilevamento di specie non falciparum, unite ai meccanism di delezione del gene pfHRP2/3 e alle dinamiche di eliminazione ritardata per $P$. falciparum nei reni, sono responsabili di un numero considerevole di falsi-positivi e falsi-negativi, in particolare per le specie non falciparum e per bassi livelli di parassitemia. ${ }^{[10,11]}$ Sono state proposte strategie per superare la delezione genica, ma la quantificazione rimane ancora una sfida. 
Un'altra famiglia di test diagnostici molto promettente è quella dei test molecolari che sfruttano meccanismi di amplificazione del DNA del Plasmodio. In particolare, i test molecolari basati sull'amplificazione isotermica del DNA ("loop mediated isothermal amplification" - LAMP), o su tecnologie simili adatte per l'uso sul campo, sono altamente sensibili $(\mathrm{LoD}=2$ parassiti/ $\mu \mathrm{l})$ ma ancora non quantitativi e rapidi, poiché richiedono un tempo di misura di circa 60 minuti. ${ }^{[12,13]}$

Altri test in fase di sviluppo sfruttano le peculiari proprietà magnetiche dei corpuscoli presenti in un campione di sangue di un paziente affetto da malaria. Nel ciclo vitale del Plasmodium, la forma mobile del parassita (sporozoita) viene iniettata nel corpo umano a seguito della puntura di una zanzara femmina della specie Anopheles e viaggia verso il fegato, dove si riproduce asessualmente dando origine a migliaia di merozoiti che infettano i globuli rossi circolanti. Il parassita inizia quindi una serie di cicli di moltiplicazione asessuata nel sangue, con un'evoluzione dei globuli rossi infetti attraverso stadi caratteristici (anello, trofozoite, schizonte), fino alla rottura della membrana cellulare e al rilascio di nuovi merozoiti che infettano altri globuli rossi. Alcuni merozoiti si sviluppano in gametociti immaturi che vengono prelevati da un'altra zanzara durante il pasto ematico, maturano nel suo intestino e danno origine a gameti femminili e maschili. Questo è l'inizio della fase di riproduzione sessuale che si svolge nel corpo della zanzara e chiude il ciclo vitale. Durante la fase asessuata intra-eritrocitaria della replicazione del Plasmodium, il parassita si nutre di emoglobina e la degrada in eme libero. Questa molecola viene convertita in una forma insolubile, portando alla formazione di cristalli nanometrici di emozoina $(\mathrm{HC})$ con suscettibilità magnetica di volume pari a $+4 \cdot 10^{-4} \cdot[14,15]$ L'emozoina è un bio-marcatore della malaria molto efficace, che si ritrova in tutti gli stadi intra-eritrocitari di tutte le specie di Plasmodium clinicamente rilevanti; ${ }^{[16]}$ per questi motivi sono stati proposti vari metodi diagnostici che si basano sul rilevamento dell'emozoina. ${ }^{[17-24]}$ Tuttavia, tali metodi non hanno finora trovato ampia diffusione sul campo. La maggior parte dei test in vitro richiede la lisi dei globuli rossi per liberare i cristalli di emozoina nella soluzione prima della misura e la quantificazione stessa della parassitemia (perecentuale di globuli rossi infetti rispetto ai sani) viene quindi effettuata in maniera indiretta.

Nel complesso manca ancora un test diagnostico rapido adatto alla quantificazione sul campo, in area endemica, di basse concentrazioni di parassiti, quali quelle che si attendono di trovare in campagne dia- 
gnostiche su popolazioni numericamente rilevanti ai fini dell'eliminazione di serbatoi per la trasmissione della malaria.

In questa nota si descrive un nuovo test diagnostico lab-on-chip sviluppato al Politecnico di Milano che sfrutta le proprietà magnetiche dei nanocristalli di emozoina. ${ }^{[25]} \mathrm{Il}$ suo nome, TMek, deriva da "Tid Mekii", il nome della malaria nella lingua locale di Mbalmayo, il villaggio del Camerun dove è stata effettuata una validazione preclinica. ${ }^{[26]}$ Su campioni di sangue con concentrazioni calibrate di globuli rossi trattati in modo da avere caratteristiche magnetiche simili a quelle dei globuli rossi infetti, il test si è dimostrato in grado di fornire una valutazione diretta e quantitativa della parassitemia in $10 \mathrm{~min}$, con una sensibilità di rilevamento fino a $10 \mathrm{i}-\mathrm{RBC} / \mu \mathrm{l}$ e un intervallo dinamico fino a $10^{5} \mathrm{i}-\mathrm{RBC} / \mu \mathrm{l}$. Il metodo fisico sviluppato per la quantificazione diretta del livello di parassitemia sfrutta la cattura magnetoforetica e la rilevazione impedenziometrica, entro un microchip, di i-RBC intatti in un campione di sangue intero. Poiché la variazione di impedenza elettrica prodotta da un i-RBC catturato tra due elettrodi è indipendente dal suo contenuto di emozoina, l'ampiezza del segnale TMek consente una valutazione quantitativa diretta della parassitemia. Si noti che, anche se la nostra prevalidazione è stata effettuata principalmente su pazienti affetti da Plasmodium falciparum, con pochi casi di $P$. ovale, $P$. malariae e P. vivax, TMek ha tutti i presupposti per diventare un test pan-malarico, poiché la trasformazione dell'emozoina in emoglobina è comune a tutte le specie di Plasmodium.

Il metodo presentato costituisce pertanto una versione automatizzata e adatta ad un utilizzo sul campo della microscopia, in quanto consente di quantificare direttamente i globuli rossi infetti. Soprattutto in aree endemiche, ciò potrebbe essere di grande aiuto nello "screening" rapido di pazienti con sospetto di malaria o nell'individuazione di portatori sani, ai fini di realizzare un efficace trattamento farmacologico personalizzato e ridurre la trasmissione dell'infezione.

\section{RISULTATI SPERIMENTALI}

\subsection{Schema del saggio diagnostico}

La forza magnetoforetica su un corpuscolo immerso in un liquido è data dalla formula $F_{\mathrm{m}}=1 / 2 \mu_{0} V \Delta \chi \nabla H^{2}$ dove $\mu_{0}$ è la permeabilità del vuoto, $\mathrm{V}$ il volume del corpuscolo, $\Delta \chi$ la suscettività relativa del corpu- 
scolo rispetto al liquido e $\nabla H^{2}$ il gradiente del quadrato del campo magnetico. I globuli rossi sani immersi in plasma hanno $\Delta \chi$ prossimo allo zero $\left(0.01 \cdot 10^{-6}\right),{ }^{[27]}$ mentre i globuli rossi infetti mostrano valori positivi che aumentano di conseguenza allo stadio del parassita a causa dell'accumulo di HC. In particolare, $\Delta \chi$ vale $0.82 \cdot 10^{-6}$ per gli anelli e $0.91 \cdot 10^{-}$ ${ }^{6}$ per i trofozoiti, forme che si possono trovare nella circolazione periferica del paziente. ${ }^{[28,29]}$ Le forme sessuate (gametociti), invece, sono costituite per quasi metà del loro volume da emozoina e quindi hanno una suscettività ben superiore $\left(\Delta \chi=2 \cdot 10^{-4}\right)$. Poiché $\mathrm{i}$ globuli rossi presentano una suscettività magnetica ben distinta nella forma sana e nei vari stati dell'infezione, è quindi possibile utilizzare la competizione fra forza gravitazionale e forza magnetica per separare i globuli rossi sani dai malati, senza utilizzare nessun apparato microfluidico attivo. I cristalli liberi di emozoina hanno suscettività relativa ancora maggiore $(\Delta \chi=$ $\left.4.1 \cdot 10^{-4}\right)$, cosicchè possono essere egualmente separati. ${ }^{[15]}$

In Fig. 1 è schematicamente rappresentata l'idea del saggio diagnostico, con riferimento a due possibili configurazioni, chiamate nel seguito per brevità "orizzontale" (Fig. 1a) e "verticale" (Fig. 1b).

In entrambi i casi il cuore del saggio diagnostico è un microchip in silicio nel quale sono realizzati 1400 microconcentratori di campo costituiti da cilindri di $\mathrm{Ni}$ (40 micron di diametro, 20 micron di altezza). In corrispondenza dei concentratori sono realizzati degli elettrodi per la misura elettrica del segnale indotto dalla cattura degli i-RBC sui concentratori. Tale microchip costituisce una delle due pareti di una camera microfluidica contenente la goccia di sangue diluito, mentre l'altra parete è un vetrino equipaggiato con opportuna guarnizione polimerica spessa 500 micron per il contenimento del campione. Quando due magneti permanenti affacciati sono posizionati in prossimità del microchip, il gradiente di campo generato dai magneti $\left(\nabla H^{2} \sim 7 \cdot 10^{14} \mathrm{~A}^{2} \cdot \mathrm{m}^{-3}\right)$ genera una forza attrattiva in tutto il volume della cella, che attrae verso la superficie del chip i corpuscoli con suscettività positiva (i-RBC e cristalli di emozoina). L'intenso gradiente localizzato alla superficie dei concentratori di $\mathrm{Ni}$, infine, permette la concentrazione dei corpuscoli in corrispondenza degli elettrodi e la massimizzazione del segnale elettrico associato.

Nella configurazione orizzontale (Fig. 1a) il microchip è posto con la faccia dei concentratori rivolto verso il basso cosicché la forza magnetica si oppone a quella gravitazionale. Dati i valori di gradiente di campo macroscopico prodotto dai magneti permanenti, in questa configurazione solo i cristalli di emozoina che hanno elevata suscettività 
$\left(\Delta \chi=4.1 \cdot 10^{-4}\right)$ riescono ad essere attratti sui concentratori, mentre tutti gli altri corpuscoli, inclusi i-RBC, sedimentano. Nella configurazione verticale, invece, la forza magnetica è ortogonale a quella gravitazionale. Tutti i corpuscoli tendono a sedimentare, ma solo i-RBC e cristalli di emozoina sono attratti verso il microchip e riescono ad essere catturati dai concentratori. Globuli rossi sani ossigenati, globuli bianchi ed altri corpuscolo sono respinti e quindi non vengono rivelati. Le due configurazioni sono quindi selettive: in quella orizzontale vengono catturati solo i cristalli di emozoina, mentre in quella verticale si catturano entrambi (cristalli di emozoina e i-RBC) ma quelli che danno maggior segnale sono gli i-RBC, a causa del loro maggior volume.
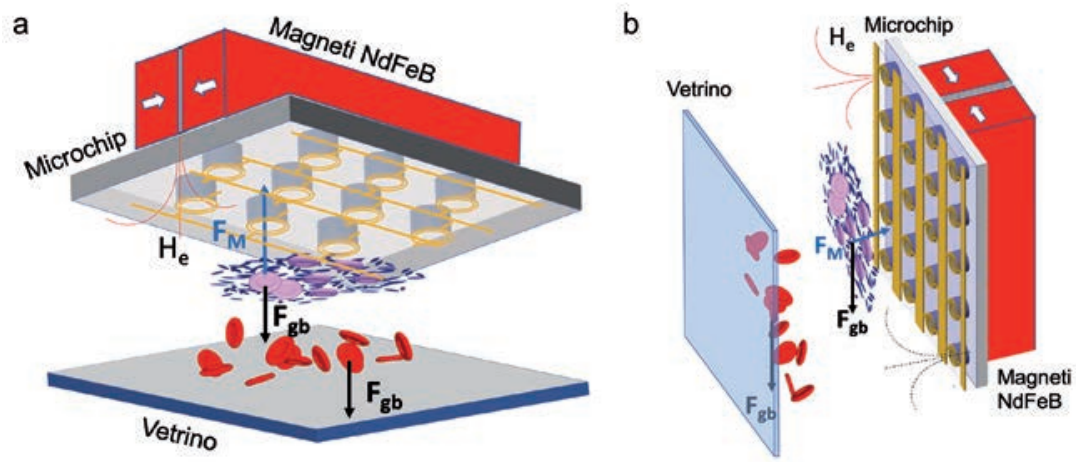

Fig. 1. Schema di funzionamento del saggio diagnostico in configurazione orizzontale (a) e verticale (b). I globuli rossi sani sono indicati in rosso, mentre quelli malati in rosa e $i$ cristalli di emozoina in nero.

In vista di un utilizzo in zone endemiche abbiamo scelto un metodo di rilevamento semplice e robusto da integrare in un microchip, che consiste in una misura differenziale di impedenza tra elettrodi anulari sopra i concentratori magnetici (elettrodi di misura, Fig. 2a) e lo stesso numero di elettrodi di riferimento, senza concentratori sottostanti. Tutti gli elettrodi sono realizzati in oro al di sopra dei concentratori di $\mathrm{Ni}$, con uno stato di $\mathrm{SiO}_{2}$ di 2 micron interposto, ed esposti direttamente al sangue. Questo schema consente un'efficiente sottrazione delle fluttuazioni spurie di modo comune. Poiché sia gli i-RBC che i cristalli di emozoina si comportano come corpuscoli isolanti nella gamma dei $\mathrm{MHz},{ }^{[15]}$ cioè nella finestra di frequenza utilizzata per rilevare le variazioni di conduttività media, la cattura è associata ad un aumento di 
impedenza (o equivalentemente diminuzione di corrente a tensione applicata di ampiezza costante) la cui ampiezza può essere correlata alla concentrazione dei corpuscoli.

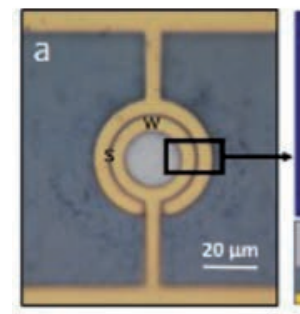

c)

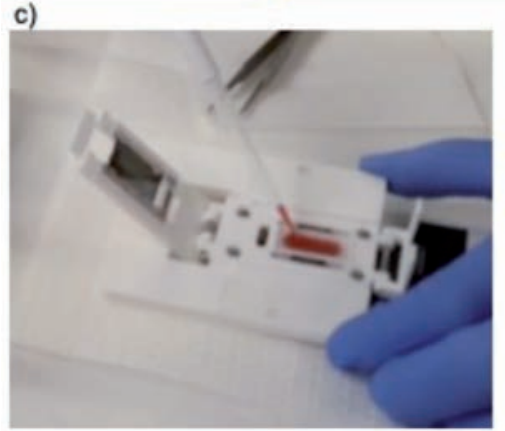

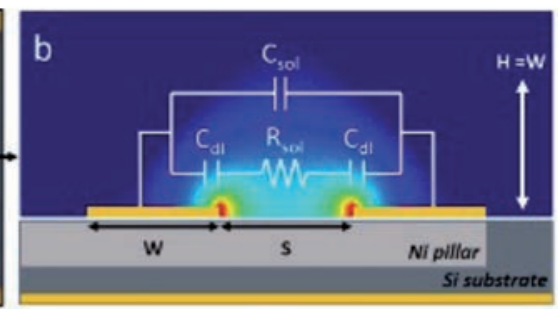

d)

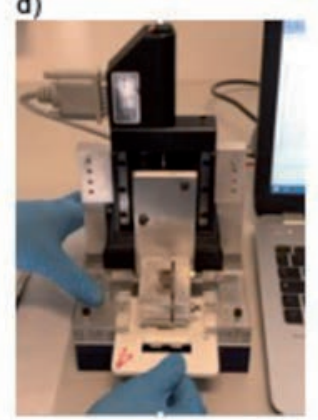

Fig. 2. a) Immagine al microscopio ottico di una coppia di elettrodi anulari in oro realizzati al di sopra di un concentratore cilindrico di Ni (cerchio grigio interno). b) Sezione degli elettrodi con larghezza $\mathrm{w}$ della pista e spaziatura s di 3 micron, con sovrapposta simulazione del campo elettrico in scala colorata e schema equivalente del circuito a parametri concentrati. c) Caricamento del campione di sangue nella cartuccia con vetrino e tasca per il microchip. d) inserimento della cartuccia nel lettore.

Il circuito elettrico equivalente del sistema elettrodi-campione è rappresentato in Fig. $2 b$ con un modello a parametri concentrati in cui sono rappresentate la capacità geometrica fra gli elettrodi mediata dalla costante dielettrica della soluzione $\left(\mathrm{C}_{\mathrm{sol}}\right)$, la capacità di doppio strato fra la soluzione e la superfice degli elettrodi $\left(\mathrm{C}_{\mathrm{dl}}\right)$ e la resistenza della soluzione $\left(\mathrm{R}_{\text {sol }}\right)$. Alla frequenza di $1 \mathrm{MHz}$ adottata negli esperimenti, una misura di impedenza è particolarmente sensibile alla resistenza della soluzione, cosicché l'interposizione fra gli elettrodi di un corpuscolo isolante determina un segnale facilmente misurabile.

In Fig. $2 c$ è rappresentata la fase di caricamento del campione entro la cartuccia in cui sono alloggiati il vetrino con la guarnizione polimerica periferica ed il microchip, infilato nella tasca di uno sportellino. Secondo 
il protocollo impiegato anche nella campagna di prevalidazione in Camerun, $8 \mu \mathrm{l}$ di sangue del paziente sono diluiti in $72 \mu \mathrm{l}$ di una soluzione di PBS (phosphate buffered saline) ed eparina (20000 IU/1) per evitare l'agglomerazione dei globuli rossi. Il campione viene quindi dispensato sul vetrino con una micropipetta e lo sportellino con il chip viene richiuso. In tal modo si sigilla la cella con il campione di sangue al suo interno e si realizzano i contatti elettrici verso una scheda elettronica mediante contatti a molla su piazzole d'oro realizzate sul chip. La cartuccia viene quindi inserita all'interno del lettore raffigurato in Fig. $2 c$, nel quale è integrato un amplificatore "lock-in" e un motore per il posizionamento dei magneti permanenti in prossimità della superficie posteriore del microchip.

\subsection{Misure su campioni infetti da Plasmodium vivax}

In Fig. 3 sono riportate le curve di corrente in funzione del tempo, registrate durante esperimenti di rilevazione di i-RBC in campioni di sangue di un paziente affetto da Plasmodium vivax e ricoverato presso il Reparto di Malattie Infettive dell'Ospedale Sacco di Milano.

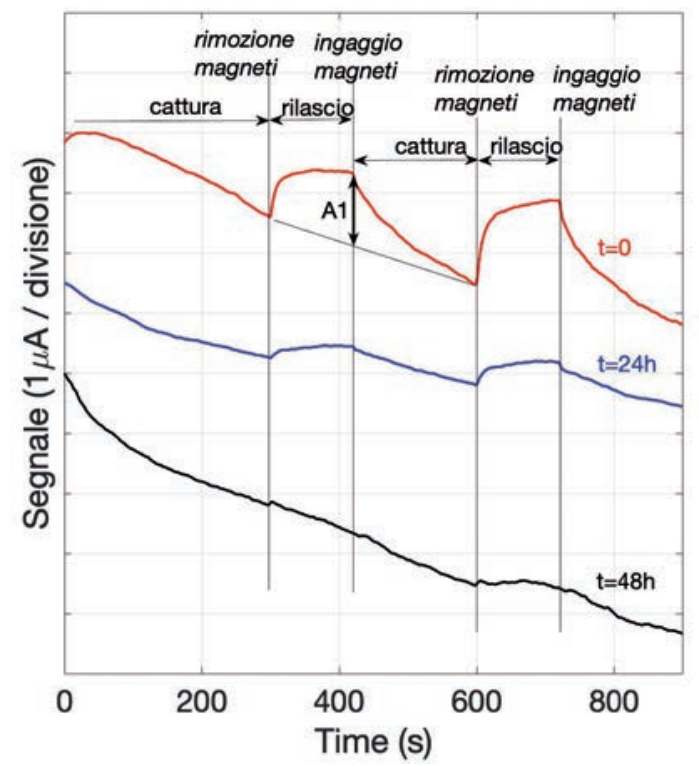

Fig. 3. Segnali differenziali di corrente a $1 \mathrm{MHz}$ di tensione alternata applicata ai capi degli elettrodi di misura e riferimento, nel caso di campioni di sangue da paziente affetto da Plasmodium vivax a tempi diversi del trattamento con clorochina: al ricovero (curva 
rossa), dopo 24 ore (curva blu) e dopo 48 ore (curva nera).

Le tre curve si riferiscono a saggi su prelievi ematici effettuati al ricovero e dopo 24 - 48 ore dall'inizio di un trattamento con clorochina. La forma caratteristica della corrente riflette il seguente protocollo di misura: (a) $t=0 \mathrm{~s}$, avvicinamento dei magneti esterni e inizio cattura; (b) $t=300 \mathrm{~s}$, allontanamento dei magneti e rilascio dei corpuscoli; (c) $t=$ $420 \mathrm{~s}$, secondo avvicinamento dei magneti e cattura; (d) t $=600 \mathrm{~s}$, allontanamento dei magneti e rilascio); (e) $t=720 \mathrm{~s}$, terzo avvicinamento dei magneti e nuova cattura. Durante la prima fase di cattura (a) la corrente diminuisce a seguito della cattura di i-RBC fra gli elettrodi, mentre a seguito del rilascio degli i-RBC indotto a $300 \mathrm{~s}$ dall'allontanamento del magnete si assiste ad una risalita della corrente. Il ciclo viene ripetuto per aumentare l'affidabilità della misura, ma l'ampiezza della prima risalita rispetto al fondo (A1) è sufficiente ad effettuare una quantificazione della concentrazione di i-RBC presenti nel campione.

Nel caso del paziente trattato in risposta ad infezione da $P$. vivax, l'analisi microscopica indicava una parassitemia iniziale dell'1\%, diminuita a circa lo $0,1 \%$ dopo 24 ore e divenuta trascurabile 48 ore dopo l'inizio del trattamento. I corrispondenti segnali TMek mostrano un'ampiezza iniziale A1 di 1 A (curva rossa), che diminuisce gradualmente fino a zero a $48 \mathrm{~h}$ (curva nera), quando un segnale residuo si vede solo in corrispondenza del secondo rilascio, a causa del tempo di cattura più lungo.

Questo esperimento preliminare dimostra le potenzialità di TMek come strumento rapido e quantitativo da utilizzare non solo per la diagnostica ma anche per monitorare l'evoluzione della malattia durante il trattamento.

\subsection{Quantificazione e limite di sensibilità}

Le capacità di quantificazione di TMek nella misura di concentrazioni di i-RBC e cristalli di emozoina in campioni di sangue sono state inizialmente valutate utilizzando modelli sintetici con concentrazioni ben note. A tale scopo, i globuli rossi di un donatore sano dell'Ospedale Sacco sono stati trattati con $\mathrm{NaNO}_{2}$ per indurre una trasformazione completa dell'emoglobina in metaemoglobina, ottenendo così globuli rossi trattati (t-RBC d'ora in poi) con comportamento magnetico simile a quello di i-RBC $\left(\Delta \chi=3.9 \cdot 10^{-6}\right)$, che sono stati poi aggiunti con varie concentrazioni ad un campione di sangue intero diluito (1:10) in PBS ed eparina per evitare l'agglomerazione. ${ }^{[30]}$ 
La Fig. 4 mostra il segnale netto, ovvero la differenza tra l'ampiezza del segnale A1 dovuta alla cattura di t-RBC e quella misurata dal campione di sangue diluito senza t-RBC (bianco), in funzione della concentrazione di t-RBC. Si osserva un comportamento lineare su più di due decadi (da $10^{2}$ a circa $5 \cdot 10^{4} \mathrm{t}-\mathrm{RBC} / \mu \mathrm{l}$, corrispondente a una parassitemia compresa tra $0,002 \%$ e $1 \%$ ) seguito da un cambiamento di pendenza a concentrazioni più elevate. Il comportamento lineare iniziale riflette pienamente la proporzionalità attesa tra la variazione di resistenza $(\mathrm{R})$ e la frazione volumetrica ( ) dei corpuscoli isolanti in prossimità di gli elettrodi, assumendo che l'efficienza di cattura sia costante. La saturazione osservata per concentrazione superiore a $10^{5}$ può essere facilmente spiegata con il completo riempimento, ad alta parassitemia, del volume sensibile al di sopra degli elettrodi da parte degli eritrociti catturati. A basse concentrazioni la curva di calibrazione di Fig. 4 mostra un limite di sensibilità di 100 $\mathrm{t}-\mathrm{RBC} / \mu \mathrm{l}$. Tale limite può essere migliorato aumentando il tempo di cattura iniziale. Ad esempio, passando da 5 a 10 minuti si ottiene un limite di sensibilità di $10 \mathrm{t}-\mathrm{RBC} / \mu \mathrm{l}$, che corrisponderebbe a 10 parassiti/ $\mu \mathrm{l}$. Tale risultato è particolarmente promettente dato che il limite di sensibilità della microscopia si attesta a circa 20 parassiti/ $\mu \mathrm{l}$ e quello dei test diagnostici rapidi attualmente in uso è dell'ordine di 200 parassiti/ $\mu$ l.

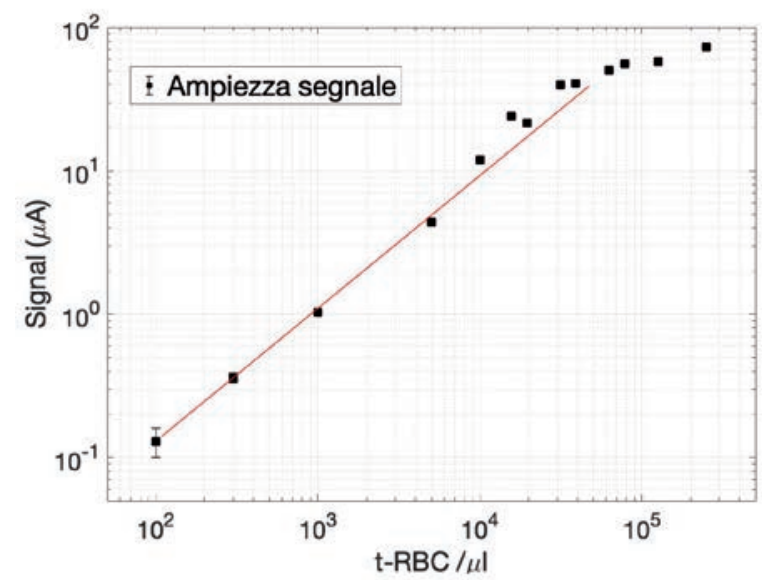

Fig. 4. Curva di calibrazione del saggio diagnostico ottenuta da campioni di sangue di donatori sani con concentrazioni note di globuli rossi trattati con $\mathrm{NaNO}_{2}(t-R B C)$ che mimano il comportamento di globuli rossi infetti da Plasmodium. E' riportata l'ampiezza del segnale A1 (vedi Fig. 3) dopo 5 minuti di cattura in funzione della concentrazione di $t-R B C$ dopo la sottrazione del segnale spurio (bianco) misurato su un campione di donatore sano senza $t-R B C$. 
Al di là del limite di sensibilità è qui opportuno però notare che la caratteristica importante del test è la possibilità di effettuare una quantificazione automatica della parassitemia, come testimoniato dall'andamento lineare su più decadi della curva di calibrazione.

La sensibilità all'emozoina libera è stata valutata utilizzando cristalli sintetici di emozoina ( $\beta$-ematina) forniti dalla ditta Invivogen, sospesi in PBS. Gli esperimenti sono stati condotti tanto nella configurazione orizzontale quanto in quella verticale, conducendo alla determinazione della medesima sensibilità. Ciò non è sorprendente poiché il gradiente di campo macroscopico prodotto dai magneti permanenti è molto più grande di quello minimo necessario per vincere la forza gravitazionale nel caso dell'emozoina, cosicchè le configurazioni verticale ed orizzontale danno segnali equivalenti. Abbiamo pertanto stimato una concentrazione minima rilevabile di $3 \mu \mathrm{g} / \mu \mathrm{l}$, che corrisponde a circa 5000 parassiti $/ \mu 1,{ }^{[17]}$ ovvero due ordini di grandezza superiore rispetto al caso di rilevamento diretto di t-RBC.

\subsection{Prevalidazione clinica}

Nell'Aprile 2019 è stata effettuata una prima campagna di validazione clinica del test in zona endemica, presso l' Hôpital Saint Luc di Mbalmayo (Camerun). E' stato condotto uno studio preclinico su 75 pazienti affetti principalmente da Plasmodium falciparum, utilizzando una classificazione di riferimento effettuata da specialisti in Malattie Infettive dell'Ospedale Sacco di Milano, sulla base di tre valutazioni indipendenti al microscopio ottico e dati clinici aggiuntivi dei pazienti. I risultati della prevalidazione sono riportati in Tabella I. Entro i limiti di questo studio preliminare con ampi intervalli di confidenza del 95\% (tra parentesi sotto), non sono emersi risultati falsi-negativi per TMek, ad indicazione dell'elevata sensibilità, 100\% (93,3-100,0), sia per campioni di sangue periferici (venosi) sia per campioni capillari. Ciò è fondamentale per evitare che un paziente affetto da malaria non sia poi effettivamente trattato con antimalarici. Nell'insieme dei campioni venosi sono stati trovati invece 9 falsi positivi, su un numero totale di 29 pazienti non affetti da malaria; la specificità del test è pertanto $69 \%(49,2-84,7)$. Al contrario, non abbiamo riscontrato risultati falsi positivi fra i campioni di sangue capillare, almeno entro il numero limitato di questi campioni (appena 10 per limiti di tempo nella prima campagna di prevalidazione). I diversi risultati ottenuti per i prelievi venosi e capillari non sono sor- 
prendenti perché i campioni venosi sono stati analizzati fino a 16 ore dopo il campionamento. In quel periodo può verificarsi una notevole degradazione dell'emoglobina, con parziale trasformazione in metaemoglobina paramagnetica che produce una suscettibilità magnetica dei globuli rossi simile a quella degli i-RBC. I campioni di sangue capillare, invece, sono stati analizzati entro 5 ore dal prelievo. Nonostante il numero limitato di campioni capillari (10) e gli ampi intervalli di confidenza dei dati in Tab. 1, i risultati preliminari ottenuti indicano che TMek potrebbe avere sensibilità e specificità molto elevate sotto forma di un "prick-test", ovvero con prelievo di sangue capillare dal dito di un paziente, come auspicabile per uno strumento diagnostico da impiegare sul campo.

Tab. 1. Risultati della prevalidazione clinica del test effettuata a Mbalmayo (Camerun).

\begin{tabular}{lcc}
\hline Risultato & TMek (venoso) & TMek (capillare) \\
\hline Veri positivi (n) & 46 & 8 \\
\hline Falsi positivi (n) & 9 & 0 \\
\hline Falsi negativi (n) & 0 & 0 \\
\hline Veri negativi (n) & 20 & 2 \\
\hline Totale test effettuati & 75 & 10 \\
\hline
\end{tabular}

\section{CONCLUSIONI}

In questa nota è stato illustrato un nuovo test diagnostico rapido per la malaria che utilizza un microchip per la separazione magnetoforetica dei globuli rossi infetti da quelli sani e la loro quantificazione mediante una misura di impedenza. Il test ha dimostrato di poter raggiungere sensibilità fino a concentrazioni di 10 parassiti/ $\mu \mathrm{l}$ di sangue buona capacità di quantificazione in un intervallo che si estende fino a $10^{5}$ parassiti/ $\mu$ l. Esperimenti preliminari su pazienti hanno permesso di seguire con il test l'evoluzione della malattia a seguito di trattamento farmacologico, verificando in modo quantitativo la decrescita della concentrazione della parassitemia. Inoltre una prima campagna sul campo, in Camerun, ha permesso di stimare i parametri caratteristici del test, pur con ampi margini di errore dovuti alla scarsa numerosità dei campioni. Sensibilità e specificità del primo prototipo sono di poco inferiori a quelle dei test rapidi per la malaria ampiamente consolidati e disponibili sul mercato. Il saggio diagnostico è ora in una fase di ingegneriz- 
zazione e caratterizzazione, al fine di realizzare una versione più affidabile e adatta ad una validazione su più ampia scala.

\section{ASPETTI ETICI}

Lo studio è stato approvato dal Comitato Etico dell'AO-Polo Universitario Luigi Sacco, Milano, Italia (Protocollo n. 17928/2018/e 12860/2019) e dal Comité Régional d'Ethique de la Recherche pour la Santé Humaine du Centre (CRERSH/C) del Camerun (CE N. 1575/CRERSHC/2019).

\section{RINGRAZIAMENTI}

R. Bertacco intende ringraziare tutti i collaboratori in questo lavoro di ricerca interdisciplinare ed in particolare: M. Giacometti, P.L. Coppadoro, G.B. Fiore e G. Ferrari del Dipartimento di Elettronica Informazione e Bioingegneria del Politecnico di Milano, F. Milesi e F. Fagiani del Dupartimento di Fisica del Politecnico di Milano, A. Rizzo della Specialità di Microbiologia e Virologia Università degli Studi di Milano, J. Bombe, M. T. Nwaha Toukam, P. F. Tina dell' Hôpital Saint Luc di Mbalmayo (Cameroon), R. Grande della UOC Microbiologia Clinica - Ospedale Sacco, S. Antinori del Dipartimento di Scienze Biomediche e Cliniche "Luigi Sacco" - Università degli Studi di Milano. La ricerca qui presentata è stata finanziata dal Politecnico di Milano, attraverso il programma "Polisocial Awards 2016" - progetto Tid Mekii, e dal programma "Switch to Product" mediante il premio "Disruptive innovation award" 2018.

\section{REFERENZE}

1. World Health Organization, Global Technical Strategy for Malaria 2016-2030 (2015).

2. Enserink, M., Eradication goal splits malaria community. Science, 365, 847-848 (2019).

3. Feachem, R.G.A. et al, Malaria eradication within a generation: ambitious, achievable, and necessary. Lancet, 394, 1056-1112 (2019).

4. World Malaria Report 2020. World Health Organization, Geneva, 2020. 
5. Van den Berg M., Ogutu B., Sewankambo N.K., Biller-Andorno N., Tanner M., RTS,S malaria vaccine pilot studies: addressing the buman realities in large-scale clinical trials. Trials, 20(1), 136 (2019).

6. Adepoju P., RTS, S malaria vaccine pilots in three African countries. Lancet, 393(10182):1685 (2019).

7. Kolluri N, Klapperich CM, Cabodi M., Towards lab-on-a-chip diagnostics for malaria elimination. Lab Chip, 18, 75-94 (2017).

8. World Health Organization. ( 2019). WHO Malaria Policy Advisory Committee ( MPAC) meeting: meeting report. April 2019. https://apps.who.int/iris/handle/10665/312198.

9. Chansuda, W., Barcus, M. J., Muth, S., Sutamihardja, A., Wernsdorfer W. H., A Review of Malaria Diagnostic Tools: Microscopy and Rapid Diagnostic Test (RDT). Am. J. Trop. Med. Hyg., 77(6), 119-127 (2007).

10. Yerlikaya, S., Campillo, A., Gonzalez, J., A systematic review: performance of rapid diagnostic tests for the detection of Plasmodium knowlesi, Plasmodium malariae, and Plasmodium ovale monoinfections in buman blood. J Infect Dis; 218, 265-76 (2018).

11. Gatton, M.L., Chaudhry, A., Glenn, J., Wilson, S., Ah, Y., Kong, A., Ord, R.L., Rees-Channer, R. R., Chiodini, P., Incardona, S., Cheng, Q., Aidoo, M., Cunningham, J., Impact of Plasmodium falciparum gene deletions on malaria rapid diagnostic test performance, Malar J, 19(1):392 (2020).

12. Illumigene Malaria LAMP, https://www.meridianbioscience.com/search/

13. Reboud J., Xu, G., Garrett, A., Yang, Z., Tukahebwa, E.M., Rowell, C., Jonathan M. Cooper, J.M., Paper-based microfluidics for DNA diagnostics of malaria in low resource underserved rural communities, PNAS, 116(11), 48344842 (2019).

14. Pagola, S., Stephens, P. W., Bohle, D. S., Kosar, A. D. \& Madsen, S. K. The structure of malaria pigment [beta]-haematin. Nature, 404, 307-310 (2000).

15. Giacometti, M., Rinaldi, C., Monticelli, M., Callegari, L., Collovini, A., Petti, D., Ferrari, G. and Bertacco, R., Electrical and Magnetic Properties of Hemozoin Nanocrystals. Appl. Phys. Lett., 113 (20) (2018).

16. Hänscheid, T., Egan, T. J., Grobusch, M. P., Haemozoin : from melatonin pigment to drug target, diagnostic tool, and immune modulator. Lancet Infect Dis. 7, 675-685 (2007).

17. Butykai, A. et al. Malaria pigment crystals as magnetic micro-rotors: key for highsensitivity diagnosis, Sci. Rep. 3, 1431 (2013).

18. Orban, A. et al., Efficient monitoring of the blood-stage infection in a malaria rodent model by the rotating-crystal magneto-optical method. Sci. Rep. 6, 23218 (2016).

19. Newman, D. M., Matelon, R. J., Wears, M. L. \& Savage, L. B., The in vivo diagnosis of malaria: feasibility study into a magneto-optic fingertip probe. IEEE J. Sel. Top. Quantum Electron. 16, 573-580 (2010).

20. Lukianova-Hleb, E. Y. et al., Hemozoin-generated vapor nanobubbles for transdermal reagent- and needle-free detection of malaria., Proc. Natl Acad. Sci. USA 111, 900-905 (2014). 
21. Scholl, P. F. et al., Rapid detection of malaria infection in vivo by laser desorption mass spectrometry. Am. J. Trop. Med. Hyg. 71, 546-551 (2004).

22. Rifaie-Graham, O. et al., Hemozoin-catalyzed precipitation polymerization as an assay for malaria diagnosis, Nat. Comm. 10, 1369 (2019).

23. Orokia Traore, Moussa Compaore, Philippe Okusa, François Hubinon, Pierre Duez, Bertrand Blankert, Martin Kindrebeogo, Development and validation of an original magneto-chromatography device for the whole blood determination of hemozoin, the paramagnetic malaria pigment, Microchemical Journal, 157, 105043 (2020).

24. Kumar, R., Verma, A.K., Shrivas, S., Thota, P., Singh, M. P., Rajasubramaniam, S., Das, A., Bharti, P. K., First successful field evaluation of new, one-minute haemozoin-based malaria diagnostic device, EClinicalMedicine 22, 100347 (2020).

25. Milesi, F., Giacometti, M., Coppadoro, P.L., Fiore, G.B., Ferrari, G., Bertacco, R., On-Chip Selective Capture and Detection of Magnetic Fingerprints of Malaria, Sensors, 20, 4972 (2020).

26. M. Giacometti et al., A lab-on-chip tool for rapid, quantitative and stage-selective diagnosis of malaria, Advanced Science, 2021.

27. Jeonghun, N., Huang, H., Lim, H., Lim, C., Shin, S., Magnetic Separation of Malaria-Infected Red Blood Cells in Various Developmental Stages, Anal. Chem. 85, n. 15 , 7316-23 (2013).

28. Moore, Lee R. et al., Open Gradient Magnetic Red Blood Cell Sorter Evaluation on Model Cell Mixtures. IEEE Trans. on Magn. 49(1), 309-15 (2013).

29. Kasetsirikul, S., Jirayut, B., Werayut, S., Morakot, K., Alongkorn, P., The Development of Malaria Diagnostic Techniques: A Review of the Approaches with Focus on Dielectrophoretic and Magnetophoretic Methods. Malaria J. 15(1),348 (2016).

30. J. Nam, H. Huang, H. Lim, C. Lim, S. Shin, Magnetic separation of malariainfected red blood cells in various developmental stages, Analytical Chemistry, 85, 7316 (2013). 
